\title{
Die EU und die Arktis: Worten Taten folgen lassen?
}

\author{
Jana Windwehr*
}

Die Region des High North von der grönländischen See bis zur Barentssee, aber auch die Arktis - verstanden als die Region nördlich des Polarkreises - insgesamt rückt zunehmend ins europäische und weltpolitische Interesse. Die Energie-, Sicherheits-, Wirtschafts- und Umweltfragen dieser Region erfordern erhöhte internationale Aufmerksamkeit, gerade unter dem Blickwinkel der Ressourcenknappheit, der Konkurrenz um Energieträger, unsicherer Produzenten- und Transitstaaten sowie des globalen Klimawandels. Die fünf Arktisanrainerstaaten - Dänemark, Kanada, Norwegen, die Russische Föderation und die Vereinigten Staaten von Amerika - haben bereits begonnen, ihre Ansprüche geltend zu machen, und es bestehen vielfältige Potenziale für Interessenkollisionen unter den Anrainerstaaten sowie mit der internationalen Gemeinschaft.

Die Europäische Union hat sich mit der Arktis-Thematik lange Zeit wenig beschäftigt; in den vergangenen Jahren scheint jedoch geradezu ein Wettbewerb zwischen den EU-Institutionen ausgebrochen zu sein, Papiere zu dieser Region vorzulegen. Die Arktispolitik der Europäischen Union ist insofern ein politikwissenschaftlich interessanter Fall, als hier die Neuentdeckung einer Region zur allmählichen Entwicklung eines neuen Betätigungsfeldes für die Europäische Union geführt hat, dieser Prozess aber bei Weitem noch nicht abgeschlossen ist. Woher rührt dieses erwachende Interesse und welche Schwerpunkte und Grundsätze haben sich als Eckpunkte einer Arktispolitik etabliert oder werden zukünftig im Zentrum stehen? Inwiefern existiert bereits eine kohärente Arktispolitik der Europäischen Union, wo sind Defizite erkennbar? Wie stellt sich die Umsetzung der Konzeptpapiere in reales Handeln , on the ground ' dar? Und worin liegen Schwierigkeiten, aber auch Gelegenheiten aus Sicht der Europäischen Union?

\section{Bedeutungswandel und -zuwachs der Arktis}

War die arktische Region während des Kalten Krieges von großer strategischer Bedeutung in der Blockkonfrontation, so hat sich in der Sichtweise der damaligen Großmächte, aber auch der Anrainerstaaten und der internationalen Gemeinschaft in den vergangenen Jahren ein Bedeutungswandel vollzogen. Sicherheitspolitische Fragen sind damit nicht von der Agenda verschwunden, sie stellen sich aber unter neuen Vorzeichen, im Vordergrund stehen die Ressourcen- und die Umweltthematik.

In der Region lagern umfangreiche Öl-, vor allem aber Gasvorkommen, die zum Teil erst kürzlich entdeckt wurden beziehungsweise durch modernste Technik und den globalen Klimawandel erst noch erschließbar werden. Eine diesbezügliche Studie des U.S. Geological Survey, ${ }^{1}$ derzufolge in der Arktis Ressourcen im Umfang von 90 Milliarden Barrel Öl und 48 Billionen Kubikmeter Erdgas lagern, ist bereits zum fixen Referenzpunkt geworden - wenngleich sie lediglich Wahrscheinlichkeiten für Rohstoffvorkommen angibt und an der Profi-

* Dr. Jana Windwehr, Martin-Luther-Universität Halle-Wittenberg.

1 Stauffer, Peter H. (Hrsg.): Circum-Arctic Resource Appraisal: Estimates of Undiscovered Oil and Gas North of the Arctic Circle, United States Geological Survey: U.S. Geological Survey Fact Sheet 2008-3049. 
tabilität des aufwändigen Abbaus für die absehbare Zukunft zum Teil Zweifel geäußert werden. ${ }^{2}$ Allerdings überlappen sich die nationalen Gebietsansprüche in den arktischen Seegebieten, da die Seegrenzen nicht überall völkerrechtlich verbindlich geregelt sind. Während die lange umstrittene russisch-norwegische Grenze in der Barentssee 2009 in einem bilateralen Abkommen festgelegt wurde, bestehen beispielsweise weiterhin unklare Grenzen zwischen Russland und den USA in der Beringsee sowie den USA und Kanada in der Beaufortsee. Folglich versuchen die Anrainerstaaten, ihre jeweilige Festlandssockel(-außen-) grenze maximal auszuweiten, wenngleich ein Großteil der Ressourcen in küstennahen und damit nicht umstrittenen Seegebieten zu finden ist. Neben Öl und Gas werden auch Blei, Diamanten, Gold, Kupfer, Silber und Zink in größerem Umfang in der Region vermutet. Trotz einer klaren formalen Regelung - bis zu zehn Jahre nach Beitritt zum Seerechtsübereinkommen (SRÜ) ${ }^{3}$ kann ein Staat die Erweiterung seiner Ausschließlichen Wirtschaftszone von 200 auf bis zu 350 Seemeilen beantragen - bestehen weiterhin Befürchtungen, Gebietsansprüche könnten auch mit illegalen Mitteln durchgesetzt werden. Neben den Energieressourcen spielt die Fischerei in der Region eine zentrale wirtschaftliche Rolle. Gerade die fischreiche Barentssee bietet Anlass zu Streitigkeiten über Fischereirechte für die Anrainersowie Drittstaaten und auf Spitzbergen bestehen neben dem Konflikt über die Regelungen des Spitzbergen-Vertrags ${ }^{4}$ von 1920 Differenzen über die Regulierung des Fischfangs. Für die gesamte arktische Region bedarf es dringend eines verantwortungsvollen Ressourcenmanagements in Bezug auf die Fischbestände.

Das Abtauen des arktischen Eises durch den globalen Klimawandel eröffnet zugleich neue Möglichkeiten beim Warentransport, zum Beispiel über den Seeweg nördlich von Russland (Nordostpassage) oder durch die nunmehr zeitweise eisfreie Nordwestpassage vor Kanada. Hier ist insbesondere zwischen Kanada und den USA umstritten, ob sie ein internes Gewässer Kanadas oder eine internationale Wasserstraße darstellt. Potenziell konfliktbeladen ist auch die Frage der maritimen Sicherheit, gerade im Zusammenhang mit Energietransporten, denn es bestehen gravierende Differenzen hinsichtlich der angestrebten Sicherheitsstandards und ihrer Umsetzung sowie Defizite in Bezug auf gemeinsame Notfallmaßnahmen und Zuständigkeitsregelungen.

Das unbestritten wichtigste Problem der arktischen Region stellt aber der Schutz der dortigen Umwelt, eng verbunden mit der Frage des globalen Klimawandels, dar. ${ }^{5}$ So trägt beispielsweise das Schmelzen des grönländischen Festlandeises nicht nur zum globalen Anstieg des Meeresspiegels bei, sondern könnte auch durch das veränderte Verhältnis von Süß- und Salzwasser potenziell Einfluss auf die Meeresströmungen, namentlich den Golfstrom, ausüben. Spätfolgen industrieller, vor allem aber auch militärischer Aktivitäten auf der russischen Seite belasten zudem die nordeuropäischen Arktisgebiete und stellen eine erhebliche

2 Vgl. beispielsweise Valur Ingimundarson: Die Kartierung der Arktis: Bodenschätze, Großmachtpolitik und multilaterale Governance, in: Aus Politik und Zeitgeschichte 5-6/2011, S. 14-23, hier S. 22.

3 Englisch United Nations Convention on the Law of the Sea (UNCLOS).

4 Treaty between Norway, The United States of America, Denmark, France, Italy, Japan, the Netherlands, Great Britain and Ireland and the British overseas Dominions and Sweden concerning Spitsbergen signed in Paris $9^{\text {th }}$ February 1920. Der Vertrag bestätigt Norwegens Hoheitsrechte, räumt aber zugleich den anderen Vertragsparteien das Recht auf ökonomische Nutzung ein, wovon derzeit lediglich Russland Gebrauch macht. Vgl. Jakub M. Godzimirski: High Stakes in the High North, Russian-Norwegian Relations and their Implications for the EU. Russia/NIS Center Russie.Nei.Visions Nr. 25/2007, hier S. 8.

5 ,[C]hanges in the Arctic sea ice cover, changes in the snow cover over Arctic land areas, glacier melting, sea level rise, changes in ocean currents, permafrost thawing, coastal erosion, and so on, are a concern not only for those who inhabit the region, but also the outside world“. Vgl. Kristian Atland: Security Implications of climate change in the Arctic, The Norwegian Defence Research Establishment (FFI): FFI-rapport 2010/01097, hier S. 24. 
Bedrohung für das dortige Ökosystem dar. Vor allem der in der Region Murmansk lagernde feste und flüssige Nuklear-Abfall ${ }^{6}$ verlangt angesichts der überbelegten, zum Teil ungeeigneten und in schlechtem Zustand befindlichen Lagerkapazitäten auch künftig nach staatenübergreifenden Anstrengungen. Auch für die Klimawandelforschung und die Entwicklung geeigneter Gegenmaßnahmen ist die Arktis als höchst sensibles Ökosystem von zentraler Bedeutung.

Nicht zuletzt hat der Klimawandel erhebliche Auswirkungen auf die Lebensweise der indigenen Völker der Arktis, exemplarisch seien hier nur Jagd und Tierhaltung genannt, Wetterbedingungen und Infrastruktur. ${ }^{7}$ Angesichts der Gefahr einer Verdrängung traditioneller Lebensweisen und -grundlagen stellen sich dringend die Fragen der Lebensqualität und der Beteiligungsmöglichkeiten der indigenen Bevölkerung.

Vor dem Hintergrund dieser Chancen und Herausforderungen haben die Anrainerstaaten des arktischen Ozeans in den vergangenen Jahren Strategiepapiere ${ }^{8}$ zur Arktis vorgelegt, und auch außerhalb der Region wächst das Interesse sichtlich. Bevor jedoch auf die Motivation der Europäischen Union eingegangen wird, sich der arktischen Region zuzuwenden, wird kurz der institutionelle Stand der Dinge zusammengefasst.

\section{Institutioneller Rahmen der Arktispolitik}

Anders als einige dramatisierende Medienbeiträge („Kalter Krieg im Hohen Norden“ etc.) in den letzten Jahren nahelegen, spielt sich der Wandel und Bedeutungszuwachs der arktischen Region nicht in einem völkerrechtlichen Vakuum ab. Grundlagendokument ist dabei zunächst das SRÜ, das die Gebietsansprüche der Küstenstaaten in Bezug auf ihre Territorialgewässer, ihre Ausschließlichen Wirtschaftszonen, ihr Kontinentalschelf und die Hohe See regelt. Im Rahmen des SRÜ ist die Festlandsockelgrenzkommission für Anträge auf Erweiterung der nationalen Gewässer eines Staates zuständig sowie die Internationale Meeresbodenbehörde ${ }^{9}$ für die Nutzung von Ressourcen der Hohen See. Allerdings verfügt das SRÜ nicht über eine Streitschlichtungsinstanz - die beispielsweise bilaterale Streitigkeiten über Seegrenzverläufe regeln könnte. Zudem haben die USA das SRÜ bis heute nicht ratifiziert.

Der Arktische Rat, in dem neben den Anrainerstaaten USA, Russland, Kanada, Norwegen und Dänemark auch Finnland, Schweden und Island vertreten sind, ist eine eher, weiche Institution, die sich vorwiegend mit dem Umweltbereich beschäftigt. Zwar weist er eine recht breite Mitgliedschaft auf und seine Arbeitsgruppen sind mit wichtigen Aspekten des Schutzes der Arktis intensiv befasst, bindende Beschlüsse trifft er gleichwohl nicht.

Mit der Erklärung von Ilulissat ${ }^{10}$ begründeten die fünf Anrainerstaaten das Gremium der Arctic Five. Sie bekennen sich in der Erklärung zu einer Lösung der offenen Fragen in der

6 Vgl. beispielsweise Ulrike Kronfeld-Goharani: Die ökologische Zeitbombe. Der Atommüll der Nordmeerflotte, in: Wissenschaft und Frieden 2/2000, S. 46-50.

7 Antje Neumann: The EU - A relevant actor in the field of climate change in respect to the Arctic?, Stiftung Wissenschaft und Politik: SWP Working Paper FG 02, 2010/03, hier S. 6.

8 Dänemark: Udenrigsministeriet: Arktis i en brydningstid, Kopenhagen 2008; Kanada: Minister of Indian Affairs and Northern Development/Federal Interlocutor for Métis and Non-Status Indians: Canada's Northern Strategy: Our North, Our Heritage, Our Future, Ottawa 2009; Norwegen: Utenriksdepartementet: The Norwegian Government's High North Strategy, Oslo 2006; Departementene: Nye byggesteiner i nord. Neste trinn i Regjeringens nordområdestrategi, Oslo 2009; Russland: Russia's Arctic Policy to 2020 and beyond, Moskau 2009; USA: White House: National Security Presidential Directive and Homeland Security Presidential Directive, Washington 2007.

9 International Seabed Authority.

10 The Ilulissat Declaration Arctic Ocean Conference Ilulissat, Greenland, 27-29 May 2008. 
Arktis im Rahmen des geltenden Völkerrechts, geben aber gleichzeitig zu verstehen, dass sie diese Fragen (insbesondere bezüglich Ressourcen) unter sich zu regeln beabsichtigen. Damit können die Arctic Five zwar ein breites Themenspektrum abdecken, sind jedoch durch den Ausschluss aller Nicht-Anrainer ein exklusiver Kreis.

Auf europäisch-regionaler Ebene existiert mit dem Barents Euro-Arctic Council (BEAC) ein Forum für intergouvernementale Kooperation, dessen Hauptziel die soziale und wirtschaftliche Entwicklung in der Region ist. Dazu kommen zahlreiche thematisch begrenzte Verträge und Regime, darunter der bereits erwähnte Spitzbergen-Vertrag von 1920, der die ökonomische Nutzung der Inseln Svalbards regelt, dessen Auslegung in Bezug auf den Kontinentalsockel aber umstritten ist. Weiterhin zu nennen sind die Guidelines for Ships Operating in Arctic Ice-covered Waters ${ }^{11}$ und der in Erarbeitung befindliche Polar Code der International Maritime Organization (IMO), die North Atlantic Marine Mammal Commission zum Schutz mariner Meeressäuger und die OSPAR-Konvention ${ }^{12}$ zur Erhaltung der Meeresumwelt des Nordostatlantiks.

\section{Interessen und Ausgangslage der Europäischen Union}

Für ein verstärktes Interesse der Europäischen Union an der arktischen Region gibt es eine Reihe von Gründen:

1. Der Arktis kommt eine wachsende wirtschaftliche Bedeutung $\mathrm{zu}$, wobei angesichts knapper und aus politisch sensitiven Regionen stammender Ressourcen insbesondere Kohlenwasserstoffe von Interesse sind. 2009 stammten beispielsweise 33 Prozent der Öl- und 34 Prozent der Gasimporte der EU-Mitgliedstaaten aus Russland, 15 beziehungsweise 30 Prozent aus Norwegen, 9 beziehungsweise 3 Prozent aus Libyen, jeweils circa 5 Prozent des Öls aus Saudi-Arabien, Kasachstan, Iran, Nigeria, Aserbaidschan und Irak, 14 Prozent des Gases aus Algerien und zwischen 2 und 4 Prozent aus verschiedenen Staaten des Nahen Ostens. ${ }^{13}$ Eine weitere Erhöhung des Anteils aus einer politisch stabilen Region im relativen Nahbereich erscheint vor diesem Hintergrund sowohl mit Blick auf die Liefersicherheit als auch die Transportkosten als attraktive Option.

2. Zukünftig neue Seeverkehrswege können durch die Einsparungen bei Energie, Kosten und Emissionen sowie die Vermeidung potenziell Piraterie-gefährdeter Gewässer (etwa am Horn von Afrika) für die außenhandelsorientierten Mitgliedstaaten der Europäischen Union von zentraler Bedeutung sein. Bisher wird die kommerzielle Nutzung zwar lediglich erprobt ${ }^{14}$ und ist mit vielen infrastrukturellen Hindernissen verbunden, sie wird aber nicht zuletzt mit Blick auf den expandierenden Asienhandel zunehmend attraktiv. Die Strecke von Hamburg nach Tokio würde sich zum Beispiel durch die Nordostpassage gegenüber der Route durch den Suezkanal um etwa 8.000 Kilometer verkürzen.

3. Die Arktis hat eine enorme Relevanz für Umwelt- und Klimafragen, nicht zuletzt in Gestalt eines weltweiten Anstiegs des Meeresspiegels, der zwar nicht vorwiegend, aber unter anderem Mitgliedstaaten der Europäischen Union betreffen wird. Die Europäische Union steht nicht nur als Verursacher eines relevanten Anteils der Verschmutzung der

11 International Maritime Organization: Guidelines for Ships Operating in Arctic Ice-Covered Waters, 23. Dezember 2002.

12 Convention for the Protection of the Marine Environment of the North-East Atlantic, text as amended on 24 July 1998, updated 9 May 2002, 7 February 2005 and 18 May 2006.

13 Daten von Eurostat: Main origin of primary energy imports, EU-27, 2001-2009.

14 Im Sommer 2009 durchfuhren beispielsweise zwei Schwergutfrachter der Bremer Reederei Beluga Shipping die Nordostpassage. 
Arktis (siehe unten) in der Verantwortung, sondern könnte in der Tat den zumindest partiell in Erscheinung tretenden Ambitionen, sich als internationaler Vorreiter in Sachen Umwelt zu etablieren, erhöhte Glaubwürdigkeit verleihen.

4. Ein weiterer Grund ist das offenkundig wachsende Interesse zum einen der Anrainerstaaten - besondere Beachtung findet das zeitweise als ,Säbelrasseln“ empfundene Verhalten Russlands ${ }^{15}$-, zum anderen weiterer externer Akteure, namentlich Chinas, aber auch der NATO. ${ }^{16}$

5. Grönlands eventuelle zukünftige Unabhängigkeit von Dänemark würde seitens der Europäischen Union neue Partnerschaftsinstrumente zur Sicherung einer langfristigen $\mathrm{Zu}$ sammenarbeit erfordern, aber auch Chancen (siehe unten) bieten.

6. Es werden Interessen der indigenen Bevölkerung berührt, die in Form der Samen auch in den nordischen Mitgliedstaaten lebt und deren Bedürfnisse folglich unter den Geltungsbereich des EU-Minderheitenschutzes fallen.

7. Möglicherweise spielt auch die internationale Präsenz der Europäischen Union eine Rolle, die hier im erweiterten Umfeld demonstriert werden könnte. ${ }^{17}$

Die Europäische Union als Nicht-Anrainer der Arktis ist allerdings, wenn sie Einfluss auf Entwicklungen in der Arktis nehmen will, weitgehend auf ihre engen Beziehungen zu Norwegen, das Teil des Europäischen Wirtschaftsraums ist, ${ }^{18}$ und zum Beitrittskandidaten Island sowie die „komplexe[...] Dreiecksbeziehung mit Dänemark und Grönland“19 nach dem grönländischen Austritt aus der Europäischen Gemeinschaft 1985 angewiesen. Sollte Island - und möglicherweise eines Tages auch Norwegen, wenngleich dies angesichts aktueller Umfragedaten auf mittlere Sicht wenig wahrscheinlich ist - Mitglied der Europäischen Union werden, könnte diese allerdings schnell ein direkter ,arktischer Player' werden. Auch deswegen ist es wünschenswert, frühzeitig eine Interessendefinition vorzunehmen und Pläne zu ihrer Umsetzung zumindest weitgehend fertig in der Schublade liegen zu wissen. Insbesondere wäre die Europäische Union vermutlich gut beraten, ihre Partnerschaft mit Grönland, das nicht nur als Rohstofflieferant, sondern eben auch als strategischer Partner und potenziell als Handels- und Transportstützpunkt interessanter wird, ${ }^{20}$ zügig weiter auszubauen. Auch die bereits bestehende enge Energiezusammenarbeit Norwegens mit der Europäischen Union insgesamt sowie mit zentralen Mitgliedstaaten, darunter Deutschland, Frankreich und Großbritannien, ${ }^{21}$ und der bestehende Energiedialog könnten durch eine vertraglich verankerte Partnerschaft weiter intensiviert werden; gegebenenfalls unter Einbeziehung Russlands, ${ }^{22}$ ähnliches gilt für die strategischen Partnerschaften mit den USA und Kanada.

Zudem verfügt die Europäische Union als Mitglied des BEAC und im Rahmen ihrer Nördlichen Dimension zumindest projektweise über einen Zugang zur arktischen Region und etablierte Kontakte zu zentralen Akteuren. Auch die nordische Zusammenarbeit in un-

15 Vgl. Godzimirski, High Stakes, 2007.

16 North Atlantic Treaty Organization.

17 Vgl. Clive Archer: An EU Arctic Policy?, Paper präsentiert auf dem Panel on EU-Russian Bilateral Relations der UACES Conference Bruges, 6-8 September 2010.

18 Dazu ausführlich: Jana Windwehr: Institutionalisierte Notlösungen oder viel versprechende Alternativkonzepte? Norwegen und die Schweiz im europäischen Integrationsprozess, Schwalbach am Taunus 2011.

19 Carsten Schymik: Grönland und Dänemark - Schlüsselländer der EU in der Arktis, in: Bernd Rill: Die Arktis. Ressourcen, Interessen und Probleme, München, 2010, S. 70-82, hier S. 70.

20 Ebenda, S. 81-82.

21 Vgl. Claudia Major/Stefan Steinicke: EU Member States' Perceptions of the Security Relevance of the High North, Stiftung Wissenschaft und Politik: SWP Working Paper FG 02/FG03-No 4-5/2011.

22 Vgl. Andreas Raspotnik: Is the European Union Missing Another Window of Opportunity for Arctic Energy Resources?, The Arctic Institute, 5.12.2011. 
terschiedlichem Rahmen könnte mittels der Mitgliedstaaten Schweden und Finnland möglicherweise eine Komponente der Arktispolitik der Europäischen Union bilden. ${ }^{23}$ Umgekehrt könnte Letztere den europäischen Anrainerstaaten bei Bedarf Unterstützung und eine bessere internationale Sichtbarkeit ihrer Positionen bieten, etwa im Rahmen der Arctic Five oder relevanter internationaler Foren.

Die Einschränkungen durch die Rolle als externer Akteur bedeuten folglich nicht, dass die Positionierung der Europäischen Union irrelevant für die weiteren Entwicklungen in der Arktis wäre. Im Gegenteil: Der weitere Verlauf der internationalen Debatte, gerade mit Blick auf weitere Verrechtlichungsprozesse, wird nicht unwesentlich von der Aufmerksamkeit und dem Einfluss wichtiger internationaler Akteure abhängen, unter denen die Europäische Union aufgrund ihrer geografischen Nähe und engen Verbundenheit zu mehreren Anrainerstaaten von zentraler Bedeutung sein könnte.

\section{Allmähliche Entwicklung einer EU-Arktispolitik}

Die Europäische Union verhielt sich lange eher abwartend gegenüber dem sich anbahnenden Bedeutungswandel und -zuwachs der Arktis, obwohl sich vor allem Norwegen um eine aktive Einbeziehung der europäischen Ebene bemühte. ${ }^{24}$ Das sogenannte Arktische Fenster innerhalb der Nördlichen Dimension existierte vorwiegend auf dem Papier, lieferte also keine greifbaren Resultate. ${ }^{25}$ In dem Papier „Climate Change and International Security“ des Hohen Vertreters für die Gemeinsame Außen- und Sicherheitspolitik und der Kommission wird erstmals explizit auf die Arktis und die möglichen zukünftigen geostrategischen/ sicherheitspolitischen Herausforderungen dort Bezug genommen. ${ }^{26}$ In der Folge begannen auch Europäisches Parlament, Kommission und Rat, sich mit der arktischen Region zu befassen. Im Herbst 2008 debattierte zunächst das Europäische Parlament arktische Fragen und legte eine Resolution vor. ${ }^{27}$ Darin wird zunächst der Hintergrund in Bezug auf das SRÜ, Ressourcenvorkommen, globale Erwärmung und deren Auswirkungen auf Mensch und Tier erläutert, um anschließend das Fehlen spezifischer, verbindlicher Regelwerke, beispielsweise für den arktischen Schiffsverkehr, zu bemängeln. Das Europäische Parlament schlägt zum einen unmittelbare Maßnahmen und kurzfristige Ziele vor, darunter die Erwähnung der Arktis auf der Kopenhagener Klimakonferenz 2009 und einen Beobachterstatus für die Kommission im Arktischen Rat, zum anderen wird als langfristige Lösung ein internationaler Schutzvertrag für die Arktis nach dem Vorbild des Antarktisvertrags vorgeschlagen. ${ }^{28}$

23 Vgl. Andreas Maurer: The Arctic Region-Perspectives From Member States and Institutions of the EU, Stiftung Wissenschaft und Politik: SWP Working Paper FG 02-4/2010.

24 So beispielsweise ein Mitarbeiter des norwegischen Außenministeriums im Interview mit der Verfasserin im November 2007.

25 Vgl. Kristine Offerdal: The EU in the Arctic: in pursuit of legitimacy and influence, in: International Journal 4/2011, S. 861-877, hier S. 865.

26 Hoher Beauftragter für die Gemeinsame Außen- und Sicherheitspolitik/Europäische Kommission: Climate Change and International Security. Paper from the High Representative and the European Commission to the European Council, S113/08, 2008.

27 Europäisches Parlament: Politisches Handeln im Arktischen Raum in einer Welt der Globalisierung. EntschlieBung des Europäischen Parlaments vom 9. Oktober 2008 zu der Politik für den arktischen Raum, in: Amtsblatt der EU, Nr. C 9 E vom 15. Januar 2010, S. 41-43.

28 Ebenda. 
Die Resolution des Europäischen Parlaments enthielt bereits einige Grundlagen für das im Folgejahr vorgelegte Arktispapier der Europäischen Kommission. ${ }^{29}$ Zentrale Ziele sind laut dem Kommissionspapier, erstens der Schutz und Erhalt der Arktis, zweitens die Förderung einer nachhaltigen Ressourcennutzung sowie drittens ein Beitrag zu einer besseren multilateralen Governance in der Region. Dabei sollen ausdrücklich anstelle neuer umfassender Vertragswerke existierende Instrumente weiterentwickelt beziehungsweise an arktische Bedingungen angepasst werden. Zudem wird einerseits gefordert, die arktischen Mitgliedstaaten in zukünftige Diskussionen einzubeziehen, andererseits soll die arktische Dimension verstärkt in Politiken der Europäischen Union Eingang finden. In institutioneller Hinsicht wird der Status eines permanenten Beobachters im Arktischen Rat als konkretes Ziel benannt. Ferner sollen Möglichkeiten grenzüberschreitender Projekte und Programme inner- und außerhalb des Rahmens der Nördlichen Dimension ausgelotet und die Idee eines European Arctic Information Centre verfolgt werden.

Aufbauend auf dem Kommissionspapier legte der Rat der Europäischen Union 2009 eigene Schlussfolgerungen vor, die beim Rat für Auswärtige Angelegenheiten am 8. Dezember 2009 angenommen wurden. ${ }^{30}$ Er unterstützt die drei genannten Hauptziele und bemüht sich stellenweise um Konkretisierung. So werden beispielsweise die arktischen Staaten aufgefordert, maritime Schutzzonen einzurichten, es wird ein temporäres Verbot von Fischerei in solchen arktischen Gewässern vorgeschlagen, die noch nicht von einem internationalen Bestandserhaltungssystem abgedeckt werden, und es wird angeregt, die überarbeiteten Arctic Council Offshore Oil and Gas Guidelines von $2009^{31}$ zu unterstützen. Auch die Bewerbung der Europäischen Kommission um permanenten Beobachterstatus im Arktischen Rat wird noch einmal unterstrichen. Für die weitere Entwicklung einer Arktispolitik der Europäischen Union legte die Europäische Kommission im Juni 2012 einen Fortschrittsbericht vor. ${ }^{32}$ In diesem plädiert sie für eine weitere Verstärkung des Engagements in der Arktis unter Beibehaltung der bisherigen Ziele. Der Bericht hält aber auch fest, dass die Europäische Union im Zeitraum seit der ersten Kommissionsmitteilung zur Arktis eine Sensibilisierung für die Auswirkungen ihrer Tätigkeit beziehungsweise der der Mitgliedstaaten auf die arktische Umwelt erwirkt und sich für eine nachhaltige Entwicklung im arktischen Raum zugunsten der lokalen Bevölkerung eingesetzt habe. Zugleich wird die Zusammenarbeit der Arktisanrainerstaaten, namentlich der Arktische Rat und das norwegisch-russische Abkommen über die Grenzziehung in der Barentssee, gelobt und eine Unterstützung dieser Kooperation seitens der Europäischen Union anvisiert. ${ }^{33}$

Die Leitziele einer EU-Arktispolitik scheinen damit im Groben abgesteckt zu sein, wobei die Auflösung des latenten Widerspruchs zwischen den ersten beiden Zielen, Umweltschutz und Ressourcennutzung, eine Herausforderung bleibt. Damit sind allerdings konkrete Schritte in der Praxis bestenfalls im Einzelfall angedacht, ein kohärenter Gesamtansatz steht weiterhin aus. Einen weiteren Schritt vorwärts in diese Richtung stellt der Bericht des Europäi-

29 Europäische Kommission: Mitteilung der Kommission an das Europäische Parlament und den Rat. Die Europäische Union und die Arktis, KOM (2008) 763.

30 Rat der Europäischen Union: Council Conclusions on Arctic Issues, 2985 ${ }^{\text {th }}$ Foreign Affairs Council meeting, Brussels, 8 December 2009.

31 Arctic Council, Protection of the Arctic Marine Environment Working Group: Arctic Offshore Oil and Gas Guidelines, 29.4.2009.

32 Europäische Kommission, Hohe Vertreterin der Europäischen Union für Außen- und Sicherheitspolitik: Entwicklung einer Politik der Europäischen Union für die Arktis: Fortschritte seit 2008 und nächste Schritte. Gemeinsame Mitteilung an das Europäische Parlament und den Rat, SWD (2012) 182/183 final.

33 Ebenda, S. 3. 
schen Parlaments ,über eine nachhaltige EU-Politik für den hohen Norden“ dar. ${ }^{34}$ Dort wird explizit auf die wachsende Aufmerksamkeit für die Arktis seitens weiterer externer Akteure, unter anderem Chinas, verwiesen. ${ }^{35}$

Als übergeordnete Ziele werden der Schutz der empfindlichen arktischen Umwelt, die Bewahrung von Frieden und Stabilität in der Region sowie die Wahrung der Interessen der dortigen Bevölkerung genannt. Die Europäische Union sei, vor allem durch ihre nördlichen Mitgliedstaaten, bereits von Entwicklungen in der Arktis betroffen und würde bei einem Beitritt Islands zukünftig Arktisanrainer. Daher betont das Europäische Parlament die „Notwendigkeit einer koordinierten Arktispolitik auf EU-Ebene" und bezeichnet die derzeitige Situation als „strategische Gelegenheit“ für eine „aktivere Rolle bei der multilateralen Governance". ${ }^{36}$ Angemahnt werden eine deutliche und transparente Prioritätensetzung, eine Benennung potenzieller Herausforderungen sowie eine kohärente Gesamtstrategie.

In diesen Positionspapieren zur arktischen Region haben sich folglich schrittweise einzelne Interessenschwerpunkte der Europäischen Union herauskristallisiert. Diese sind allerdings nicht mit einer eindeutigen Priorisierung versehen und zunächst eher als allgemeine Leitlinien denn als konkrete Operationalisierungen von Interessen zu verstehen. Eine kontinuierliche, quasi-lineare Entwicklung einer kohärenten Arktispolitik, die mit einem stetig steigenden Interesse der Europäischen Union, ihrer Institutionen und Mitgliedstaaten einherginge, lässt sich aus dieser Entwicklung bisher nicht ableiten. ${ }^{37}$

Eine gemeinsame Position der Europäischen Union zur arktischen Region insgesamt existiert bisher nicht, vielmehr scheint nach anfänglichem Zögern ein gewisser institutioneller Wettbewerb um die Vorlage von Strategiepapieren und damit möglicherweise um die Deutungshoheit und Zuständigkeit in dieser Frage eingesetzt zu haben. Die Arktispolitik reflektiert insofern - wenig überraschend - die allgemeine Problematik einer intergouvernemental organisierten und institutionell zersplitterten Gemeinsamen Außen- und Sicherheitspolitik (GASP). Die Akteursvielfalt aus Kommission und ihren Generaldirektionen, ${ }^{38}$ Rat der Europäischen Union und Europäischem Parlament, seit dem Vertrag von Lissabon ergänzt um die Hohe Vertreterin der Union für Außen- und Sicherheitspolitik (Hohe Vertreterin) samt den Europäischen Auswärtigen Dienst, erschwert die Herausbildung eines kohärenten Ansatzes oder führt zumindest dazu, dass sich dieser nur über einen längeren Zeitraum entwickeln kann. Der Vertrag von Lissabon schafft durch den ,Doppelhut ${ }^{6}$ der Hohen Vertreterin als Vorsitzende des Rates für Auswärtige Angelegenheiten und Vizepräsidentin der Europäischen Kommission insofern zumindest günstigere Bedingungen, dieses komplexe Institutionen- und Akteursgefüge besser zu koordinieren. Inwiefern dies tatsächlich gelingt, lässt sich derzeit zumindest in Bezug auf die Arktis noch nicht seriös bewerten.

Dass die Mitgliedstaaten ${ }^{39}$ erheblich unterschiedlich großes Interesse an der Region zeigen, ist ebenfalls kein Spezifikum der Arktis-Frage, erschwert aber die Herausbildung einer gemeinsamen Linie zusätzlich. Selbsterklärend ist hier zunächst das erhebliche Interesse der nordischen EU-Staaten Schweden und Finnland an regionalen Fragen beziehungsweise

34 Europäisches Parlament: Nachhaltige Politik der EU für den hohen Norden. Entschließung des Europäischen Parlaments vom 20. Januar 2011 zu einer nachhaltigen EU-Politik für den hohen Norden, P7_TA(2011)0024.

35 Ebenda, S. 6.

36 Europäisches Parlament: Nachhaltige EU-Politik, 2011, S. 8.

37 Vgl. Offerdal: EU in the Arctic, 2011, S. 862.

38 In den Themenbereich fallen beispielsweise Zuständigkeiten der Generaldirektionen für Energie, Klimapolitik, maritime Angelegenheiten und Umwelt.

$39 \mathrm{Zu}$ Deutschlands Interessen und Ausgangslage: Helga Haftendorn: Zaungast in der Arktis, in: Internationale Politik 4/2011, S. $72-79$ 
Rückhalt durch die Europäische Union, während für andere Mitgliedstaaten ganz andere regionale Zusammenhänge Priorität haben. Zudem wählen einzelne Mitgliedstaaten zur Vertretung ihrer Interessen nicht notwendigerweise den Weg über die gemeinsame EU-Position: So zählt zum Bewerberkreis für den Status eines ständigen Beobachters im Arktischen Rat neben mehreren asiatischen Staaten und der Europäischen Union auch Italien. Inwiefern einzelne Mitgliedstaaten bereit sind, ihre arktischen Interessen im Rahmen einer gemeinsamen EU-Arktispolitik zu vertreten, wird in erheblichem Maße davon abhängen, ob es gelingt, eine solche kohärente Politik auf EU-Ebene zu etablieren. Mit anderen Worten: je weniger koordiniert und sichtbar die Position der Europäischen Union, desto größer die Wahrscheinlichkeit nationaler ,Alleingänge` und anhaltender Widersprüche in dieser Frage. Der Folgeabschnitt richtet den Blick auf die drei nunmehr etablierten Zielvorstellungen und versucht, eine - zugegebenermaßen auf einen kurzen Zeitabschnitt bezogene - Bilanz der Umsetzung beziehungsweise der Aktivitäten in diesen drei Bereichen zu ziehen.

\section{Aktivitäten und zukünftige Betätigungsfelder}

\section{Umweltschutz}

Der Klimawandel in der Arktis wird überwiegend von Faktoren außerhalb verursacht. Wie das „Arctic Footprint Assessment“ bestätigt, trägt die Europäische Union beziehungsweise tragen ihre Mitgliedstaaten unter anderem durch Chemikalien und Treibhausgase zum arktischen Klimawandel bei. ${ }^{40}$ Das wird zwar seitens der Europäischen Union durchaus als Tatsache anerkannt, dennoch hat die Arktis im Rahmen der Umweltpolitik der Europäischen Union bisher kaum eine nennenswerte Rolle gespielt. Im europäischen Nahbereich sind allerdings Projekte, zum Teil mit Beteiligung der Europäischen Union oder einzelner Mitgliedstaaten, zur nuklearen Sicherheit und der Bewältigung von Altlasten im russischen Nordwesten zu nennen. Im Rahmen der integrierten Meerespolitik der Europäischen Union findet die Arktis zwar Erwähnung, was sich aber bisher nicht in konkreten Maßnahmen niedergeschlagen hat. Dieser Befund ist symptomatisch für die EU-Umweltpolitik für die Arktis insgesamt, die bisher einen weitgehend deklaratorischen Charakter aufweist. ${ }^{41}$

Allerdings würde die Arktis in erheblichem Maße von wirksamen globalen Klimaschutzabkommen profitieren, wenngleich sie nicht explizit beziehungsweise nur am Rande von diesen in den Blick genommen wird. ${ }^{42}$ Hier engagiert sich die Europäische Union durchaus in erheblichem Maße, beispielsweise durch ihren Einsatz für ein verbindliches Kyoto-Folgeabkommen. Der Fortschrittsbericht der Kommission hebt das Engagement der Europäischen Union bei der Senkung der Treibhausgasemissionen hervor. ${ }^{43} \mathrm{Da}$ ein ambitioniertes, bindendes Klimaabkommen bestenfalls langfristig realistisch sein dürfte, sind allerdings kurzfristige regionale Maßnahmen unerlässlich. Die genannte Entschließung des Europäischen Parlaments schlägt dazu vor, verstärkt mit dem Rahmenübereinkommen der Vereinten Nationen UNFCCC ${ }^{44}$ und $\mathrm{SAON}^{45}$ zusammenzuarbeiten, weiterhin werden eine Einbezie-

40 Vgl. Sandra Cavalieri et al.: Spurensuche: Der ökologische Fußabdruck der EU in der Arktis, in: Osteuropa 2-3/2011, S. 211-223.

41 Vgl. auch Neumann: A relevant Actor, 2010, S. 30.

42 Vgl. ebenda, S. 18-19.

43 Vgl. Europäische Kommission: Fortschrittsbericht, 2012, S. 4 und 14.

44 United Nations Framework Convention on Climate Change.

45 Sustaining Arctic Observing Networks. 
hung der Europäischen Umweltagentur und die Regulierung von Rußemissionen gefordert. $^{46}$

Im Bereich der Klimaforschung dagegen zeigt die Europäische Union bereits heute einen deutlich höheren Aktivitätsgrad: So wurde innerhalb des Siebten Forschungsrahmenprogramms zum Beispiel das Projekt Arctic Tipping Points ins Leben gerufen, das besonders sensible Elemente des arktischen Ökosystems als Indikatoren für Klimawandelprozesse in den Blick nimmt. Im Rahmen eines weiteren Projekts, ArcRisk, werden die gesundheitlichen Auswirkungen des Klimawandels auf die Bevölkerung der Arktis und weiterer europäischer Gebiete untersucht. ${ }^{47}$ Insgesamt hat die Europäische Union im Rahmen des Siebten Forschungsrahmenprogramms etwa 200 Millionen Euro für Forschungsaktivitäten in der Arktis zur Verfügung gestellt. ${ }^{48}$ Zur weiteren Stärkung der wissenschaftlichen Zusammenarbeit mit den Anrainerstaaten liegt weiterhin der oben genannte Vorschlag zur Schaffung eines Informationszentrums EU-Arktis auf dem Tisch. Zusammenfassend ist folglich bisher eine Konzentration auf globale Klimaverhandlungen einerseits und den Forschungsbereich andererseits zu konstatieren, während lokale und regionale Maßnahmen nur punktuell eine Rolle spielen.

\section{Nachhaltige Ressourcennutzung}

Relativ wenig konkret stellt sich auch die Position der Europäischen Union im Hinblick auf die nachhaltige Nutzung arktischer Ressourcen dar. Der Europäischen Union bleibt keine andere Möglichkeit, als die souveränen Rechte der Anrainerstaaten anzuerkennen und durch den eigenen Status als wichtiger Verbraucher, Ursprung großer Wirtschaftsunternehmen und eventuell als Exporteur relevanter Technologien Einfluss auszuüben. Der zuvor erwähnte, vom Europäischen Parlament verabschiedete Bericht fordert in diesem Sinne für die Förderung arktischer Ressourcen höchstmögliche Sicherheits-, Sozial- und Umweltstandards, einen ökosystembasierten Gesamtansatz, Umweltverträglichkeitsprüfungen und Abschätzungen strategischer und sozialer Auswirkungen. Aufgrund der Größe ihres Marktes und des bereits bestehenden Ausmaßes der Öl- und Gasimporte namentlich aus Russland und Norwegen (siehe oben) stellt sich weniger die Frage, ob die Europäische Union Zugang zu den arktischen Ressourcen erhalten wird, sondern in welchen Mengen, durch welche Transportkanäle und welche Möglichkeiten bestehen werden, die Förderbedingungen aktiv zu beeinflussen.

Mit einem großen Fragezeichen hinsichtlich der Möglichkeiten einer Einflussnahme zu versehen ist der Aspekt der Nachhaltigkeit. Die anvisierte Unterstützung der überarbeiteten Leitlinien des Arktischen Rates für die Offshore-Erdöl- und -Erdgasförderung ist sicherlich ein Baustein. Mit Blick auf andere Ressourcen bietet die Regulierung der Fischerei in Teilen der Region ein weiteres Betätigungsfeld, auf dem die Europäische Union sich beispielsweise für einen verbesserten Bestandsschutz oder geschützte Gebiete einsetzen könnte. ${ }^{49}$

Die offenen Grenz- und Kontinentalsockelfragen sind von den Anrainerstaaten untereinander unter Berücksichtigung der völkerrechtlichen Grundlagen zu klären. Allerdings könnte sich die Europäische Union in ihrem Nahbereich für ein Zusatzabkommen zum Spitzbergen-

46 Europäisches Parlament: Nachhaltige EU-Politik, 2011, S. 11-12.

47 Nähere Informationen zu den Projekten sind auf den jeweiligen Websites abrufbar unter: www.eu-atp.org beziehungsweise www.arcrisk.eu.

48 Vgl. Europäische Kommission: Fortschrittsbericht, 2012, S. 4.

49 Vgl. zur Fischereifrage Bettina Rudloff: Fisch im Wasser? Die EU und die Arktisfischerei, in: Osteuropa 2-3/2011, S. 163-178. 
Vertrag - zu dessen Unterzeichnerstaaten auch eine Reihe von Mitgliedstaaten zählt - einsetzen, das den Status des Kontinentalsockels der Inseln regeln würde; bisher sind allerdings keine diesbezüglichen Bemühungen erkennbar.

\section{Bessere Governance}

Als drittes Ziel des Kommissionspapiers wird eine verbesserte Governance-Architektur für die Arktis benannt. Zwar besteht kein rechtliches Vakuum, doch die bestehende Rechtslage, geprägt durch das SRÜ und eine Vielzahl thematisch und/oder regional begrenzter Abkommen, weist durchaus einige Regelungslücken im Sinne von ,governance gaps ‘ (fehlende oder unzureichend ausgestattete Institutionen) beziehungsweise ,regulatory gaps (nicht oder unzureichend geregelte Sachbereiche) auf. ${ }^{50}$ Neben der medienwirksamen, aber faktisch nur für einen relativ kleinen Anteil strittigen Frage nach den Besitzverhältnissen der arktischen Rohstoffe betrifft dies vor allem die umweltverträgliche Förderung arktischer Ressourcen sowie die Regulierung der Schifffahrt in der Region.

Für die Schifffahrt stellt sich etwa zum einen die Frage nach den Durchfahrtsrechten durch die Nordost- und Nordwestpassage, wobei die Europäische Union die gleiche Position einnimmt wie die USA, dass nämlich beide Passagen internationale Wasserstraßen darstellen, durch die eine freie Durchfahrt gewährleistet sein muss. Kanada und Russland dagegen vertreten die Position, es handele sich vor allem aus historischen Gründen um nationale Gewässer. Diese Differenzen haben zwar bisher nicht zu größeren Konflikten geführt; gleichwohl ist man einer völkerrechtlichen Lösung auch noch nicht erheblich näher gekommen. Die Europäische Union, die mit ihren Mitgliedstaaten 40 Prozent der weltweiten Handelsschifffahrt kontrolliert, ${ }^{51}$ hat ein großes Interesse, hier zu einer verbindlichen Regelung zu finden, hat sich bisher aber nicht in dieser Frage positioniert. Zugleich würde eine verstärkte Nutzung insbesondere im Fall der Nordostpassage erhebliche Investitionen in die Hafen-, Landanbindungs- und Sicherheitsinfrastruktur erforderlich machen, wobei die Europäische Union beziehungsweise einzelne Mitgliedstaaten und europäische Unternehmen möglicherweise ebenfalls eine Rolle spielen könnten. Eine wirtschaftliche Nutzung der arktischen Seeverkehrswege ist aber eine eher langfristige Option, die derzeit unter anderem aufgrund unzureichender Infrastruktur und ungeklärter Versicherungsfragen bestenfalls zu experimentellen Zwecken genutzt wird.

Zum anderen weist der Bereich Schifffahrt natürlich enge Verbindungen zur Umweltthematik auf. Hier gilt es, eine bessere Koordinierung und mittelfristige Angleichung der nationalen Rechtsvorschriften im Bereich der arktischen Schifffahrt zu erwirken, nicht zuletzt vor dem Hintergrund eines stetig wachsenden touristischen Schiffsaufkommens in der Region. Die Europäische Union beteiligt sich bereits an den Arbeitsgruppen des Arktischen Rates, namentlich zu Search-and-Rescue-Aktivitäten, und unterstützt die Arbeit der IMO an einem verbindlichen Polarkodex.

Neben diesen die Arktis insgesamt betreffenden Fragen können allerdings auch regionale Arrangements eine Rolle für die zukünftige Governance-Architektur in der Region spielen. Hier ist neben den bereits erwähnten Einrichtungen BEAC und Nördliche Dimension auch hervorzuheben, dass die Europäische Union als Teil des Nachbarschafts- und Partnerschafts-

50 Timo Koivurova/Erik J. Molenaar: International Governance and Regulation of the Marine Arctic - Overview and Gap Analysis. WWF International, Arctic Programme, 2009, hier S. 5.

51 Vgl. Europäisches Parlament: Nachhaltige EU-Politik, 2011, S. 8. 
instruments (ENPI) mit Kolarctic und KARELIA zwei Projekte grenzüberschreitender Zusammenarbeit fördert. ${ }^{52}$

Eine oft vernachlässigte Dimension der arktischen Governance stellen die in der Arktis lebenden indigenen Völker dar. Sie sind nicht nur in erheblichem Maße vom Klimawandel und den Chancen und Risiken zukünftiger Ressourcennutzung betroffen, sondern es gilt zugleich, diese Gruppen mit ihren spezifischen Bedürfnissen, aber auch ihren Kenntnissen und ihrem traditionellen Wissen über die Region in die Problemlösung einzubinden. Die Europäische Union hat in der Vergangenheit mehrfach ihre Rechte anerkannt, ${ }^{53}$ insbesondere mit Blick auf den Status der Samen beim Beitritt Schwedens und Finnlands. Zudem werden die indigenen Völker in die Gespräche zu lokalen und regionalen Fragen im Rahmen der Nördlichen Dimension eingebunden. ${ }^{54}$ Die Politik der Europäischen Union zielt auf eine Stärkung ihrer wirtschaftlichen Lebensgrundlagen, den Erhalt ihrer Kultur, einen Beitrag dieser Gruppen zum Erhalt der Biodiversität in der Region beispielsweise durch nachhaltige Ressourcennutzung sowie eine verbesserte Zusammenarbeit durch Dialogformate. Von verschiedenen Förderinstrumenten wie beispielsweise dem Fonds für Regionale Entwicklung (EFRE), dem Botnia-Atlantica-Programm sowie dem Programm für die Nördlichen Randgebiete profitiert unter anderem auch die indigene Bevölkerung. ${ }^{55}$ Eine Stärkung der Kooperation mit den arktischen Gemeinschaften und die Unterstützung für deren Position bieten der Europäischen Union eine Chance, sich aktiv in regionale Fragen einzubringen und zugleich für einen partizipativen - anstelle eines exklusiven - Gesamtansatz der arktischen Governance zu werben.

\section{Chance und Bewährungsprobe für die GASP, herausfordernde ,Beziehungsarbeit`}

Die Europäische Union steht damit im Jahr 2012 vor einer doppelten Herausforderung: Zunächst müssen die benannten Ziele und in Teilen bereits angedachten Mittel koordiniert und zu einem Gesamtansatz inklusive einer präzisen Definition und Prioritätensetzung der EU-Interessen zusammengefügt werden. Die Europäische Kommission konstatiert selbst, dass die bisherigen Maßnahmen in den Bereichen Umweltschutz, Forschung und wirtschaftliche Entwicklung zwar zu einigen ,greifbaren Ergebnissen“ geführt hätten, es jedoch nötig sei, den politischen Kurs in der Region ,zu präzisieren, einen umfassenderen Ansatz zu verfolgen und diesen mit der Agenda Europa 2020 für intelligentes, nachhaltiges und breitenwirksames Wachstum zu verknüpfen". ${ }^{56}$ Kernelemente sollen dabei unter dem Slogan „Wissen, Verantwortung und Engagement“ Investitionen in die Forschung, Maßnahmen für nachhaltiges Wirtschaften sowie eine enge Zusammenarbeit mit Anrainerstaaten, relevanten Institutionen und indigener Bevölkerung sein. ${ }^{57}$

Erschwerend wirken sich dabei die bekannten Schwierigkeiten der intergouvernemental organisierten GASP aus: eine Vielzahl von Akteuren, die die Herausbildung kohärenter Positionen erschwert, teilweise divergierende Interessen und Handlungspräferenzen der Mit-

52 Nähere Informationen zu den Projekten sind abrufbar unter www.kolarcticenpi.info beziehungsweise www.kareliaenpi.eu.

53 Vgl. im Einzelnen Neumann: A Relevant Actor, 2010, S. 9-10.

54 Vgl. Europäische Kommission: Mitteilung der Kommission an das Europäische Parlament und den Rat. Die Europäische Union und die Arktis, KOM (2008) 763.

55 Eine Auflistung der hier relevanten Förderprogramme findet sich im Fortschrittsbericht der Europäischen Kommission, 2012, S. 15-16.

56 Europäische Kommission: Fortschrittsbericht, 2012, S. 5.

57 Vgl. ebenda, S. 6-13. 
gliedstaaten, die häufig daraus resultierende Einigung auf den kleinsten gemeinsamen Nenner. In dieser Hinsicht kann die Arktispolitik möglicherweise tatsächlich einen „stern test for European maturity “58 darstellen. In der Arktis besteht die Chance, eine Dimension der GASP längerfristig zu entwickeln, ohne permanent auf dramatische Ereignisse reagieren zu müssen wie an den zahlreichen internationalen Krisenherden. Die bisherige Entwicklung war allerdings stark von interessierten Einzelpersönlichkeiten und medienwirksamen Ereignissen wie dem vielzitierten Platzieren einer russischen Flagge auf dem arktischen Meeresboden abhängig und der Europäische Auswärtige Dienst hat sich noch nicht allzu sichtbar in diesem Themenbereich profiliert. ${ }^{59}$ Mit dem Besuch der Hohen Vertreterin in den nordeuropäischen Arktisanrainerstaaten 2012 sowie der Vorlage des bereits erwähnten Fortschrittsberichts deutet sich möglicherweise allerdings auf mittlere Sicht eine erhöhte Aufmerksamkeit und Priorität der Region auch im Europäischen Auswärtigen Dienst an.

In den betrachteten Papieren wurden allgemeine Grundsätze etabliert, die jedoch nur zu geringen Teilen von konkreten Handlungsvorschlägen flankiert wurden. Die 2011 verabschiedete Entschließung des Europäischen Parlaments geht in dieser Hinsicht allerdings einen Schritt weiter als die vorangegangenen Dokumente und auch der Fortschrittsbericht der Europäischen Kommission zeigt, dass zunehmend greifbare Maßnahmen durchgeführt oder anvisiert werden. Zudem ist die Formulierung von Erwartungen an die Arktisanrainerstaaten, so zum Beispiel hinsichtlich einer Stärkung der Grundlagen und Kompetenzen des Arktischen Rates, sicherlich legitim und angesichts deren privilegierten Entscheidungsstatus' sinnvoll, dennoch wird es nicht ausreichen, anstelle eigener Initiativen allein auf die Erfüllung dieser Erwartungen zu setzen. Vielmehr ist die Europäische Union einerseits darauf angewiesen, die Nördliche Dimension und ihre Mitgliedschaft in relevanten regionalen (BEAC) und internationalen Organisationen (IMO und andere) für problemspezifische Einflussnahme zu nutzen, andererseits einzelne Mitglied- und Partnerstaaten als Informations- und Einflusskanäle zu nutzen. Namentlich die Mitgliedstaaten Dänemark, Schweden und Finnland sowie Norwegen und Island als Mitglieder des Europäischen Wirtschaftsraums sowie eine engere Zusammenarbeit mit Grönland dürften dabei eine wesentliche Rolle spielen. Während Dänemark quasi ,zwischen den Stühlen“ sitzt und seine arktischen Interessen besser im Rahmen der Arctic Five denn durch die Europäische Union vertreten kann, ${ }^{60}$ gilt im Umkehrschluss für Finnland, Schweden und Island, die dort nicht vertreten sind, dass sie eine stärkere Rolle der Europäischen Union aller Voraussicht nach unterstützen werden. Im isländischen Fall ist zudem die Beitrittsfrage von erheblicher Bedeutung für die Arktispolitik der Europäischen Union; allerdings steht die Arktis umgekehrt nicht im Zentrum der isländischen Beitrittsdebatte. Mit den anderen relevanten Akteuren allerdings hat die Europäische Union noch langwierige und intensive ,Beziehungsarbeit‘ zu leisten, insbesondere mit Russland und in der Beobachterstatus-Frage auch mit Kanada. ${ }^{61}$

In den transatlantischen Beziehungen, in denen die arktische Region bisher kaum eine Rolle gespielt hat, wäre durchaus eine Themenerweiterung in diese Richtung denkbar. Die

58 Alyson Bailes: How the EU Could Help Cool Tempers over the Arctic, in Europe's World, 19.6.2009.

59 Vgl. Offerdal: EU in the Arctic, 2011, S. 874. In seiner Entschließung schlägt das Europäische Parlament in diesem Sinne vor, die Kommission solle die bestehende ,inter-service group on Arctic in eine ständige Struktur umwandeln, die gemeinsam durch EAD und GD Mare geleitet werden könnte; zudem wird die Einrichtung eines Arktis-Referats innerhalb des EAD angeregt. Vgl. Europäisches Parlament: Nachhaltige EU-Politik, 2011, 16-17.

60 Malte Humpert: EU Arctic Policy: A Memorandum to the European Commission, The Arctic Institute, Mai 2012.

61 Offerdal: EU in the Arctic, 2011, S. 863. 
Erfahrungen vor allem Norwegens beispielsweise bei der Bewältigung der desolaten ökologischen Zustände auf der Kola-Halbinsel, aber auch im Hinblick auf die Erschließung des Stockmann-Feldes ${ }^{62}$ zeigen darüber hinaus, dass die arktische Kooperation mit Russland zwar nicht überall reibungslos verläuft, aber doch Früchte tragen kann.

Als zentraler Grund für Kanadas ablehnende Haltung gegenüber einem permanenten Beobachterstatus der Europäischen Union im Arktischen Rat wird offiziell das EU-Verbot des Handels mit Seehundprodukten angeführt. Unabhängig von der zukünftigen Entwicklung dieser Blockadehaltung muss allerdings ohnehin einschränkend bemerkt werden, dass ein Beobachter im Arktischen Rat eben ein Mitglied ohne Stimmrecht in einer Organisation wäre, die zwar im Umweltbereich bereits Erhebliches geleistet hat, jedoch bis auf Weiteres eine ,weiche“ Institution mit eingeschränkter Zuständigkeit bleibt.

Dagegen erscheint es durchaus als realistische und erfolgversprechende Option, dass die Europäische Union in Bezug auf einzelne thematische Regime ihr internationales Gewicht in die Waagschale wirft. Andreas Maurers Vorschlag, die Europäische Union solle sich international für den Schutz gemeinsamer, universaler Interessen in der Arktis, darunter Umweltschutz, Frieden und Sicherheit und die Bedürfnisse der indigenen Bevölkerung einsetzen, ${ }^{63}$ ist sicherlich zuzustimmen. Malte Humperts Forderung dagegen, die Europäische Union solle trotz der absehbaren Aussichtslosigkeit des Vorhabens für einen möglichst umfassenden Vertrag à la Antarktis-Vertrag eintreten, um sich international zu profilieren, ${ }^{64}$ wird mit großer Sicherheit zu Abwehrreaktionen bei den Anrainerstaaten führen - und könnte damit letztendlich einer effektiven EU-Arktispolitik abträglich sein.

Die sicherheitspolitische Dimension schließlich wurde bisher seitens der Europäischen Union wenig thematisiert und wird sicherlich auch in Zukunft keine zentrale Rolle spielen, wenn nicht unvorhergesehene Ereignisse und eine dramatische Verschärfung der Konflikte in der Arktis dies erforderlich machen. Angesichts des gemeinsamen Interesses der Anrainerstaaten, die offenen Ressourcen- und Grenzfragen im Einklang mit dem Völkerrecht zu regeln, ist ein größerer, mit militärischen Mitteln ausgetragener Konflikte nach heutigem Stand wenig wahrscheinlich; dennoch ist die Europäische Union sicher gut beraten, auch die zum Teil erheblichen Rüstungsanstrengungen der Anrainer und insbesondere die Situation ihrer Mitglieds- beziehungsweise Partnerstaaten genau zu beobachten.

62 Das Stockmann-Feld ist ein Gasfeld in der Barentssee.

63 Maurer: The Arctic Region, 2010, S. 18.

64 Humpert: EU Arctic Policy, 2012, S. 29-30. 